\title{
Intervenção Coronariana Percutânea em Pacientes com Mais de 70 Anos: Análise de Desfechos Intra-Hospitalares na Era Contemporânea
}

\author{
Cristiano de Oliveira Cardoso ${ }^{1}$, Carlos Roberto Cardoso ${ }^{1}$, La Hore Correa Rodrigues ${ }^{1}$, Clacir Staudt $^{1}$, \\ Aldo Fernando Somavilla Duarte', Vasco Morosini Miller ${ }^{1}$, Michelle de Oliveira Cardoso ${ }^{1}$, \\ La Hore Correa Rodrigues Jr. ${ }^{1}$, Luis Maria Cabreira Yordi ${ }^{1}$
}

\section{RESUMO}

Introdução: Dados observacionais da década de 90 sugerem que pacientes com mais de 70 anos de idade evoluem com maior risco de eventos quando submetidos a intervenção coronariana percutânea (ICP). Objetivo: Avaliar os desfechos hospitalares em pacientes com idade superior a 70 anos submetidos a ICP na era contemporânea. Método: Entre janeiro de 2005 e junho de 2007, pacientes submetidos a ICP foram incluídos consecutivamente em um registro. Informações referentes a fatores de risco, indicação do exame, detalhes técnicos da ICP e desfechos intra-hospitalares foram prospectivamente coletadas. Os pacientes foram divididos em dois grupos: idade $<$ ou $>70$ anos. Para comparação, foram utilizados os testes $t$ de Student e qui-quadrado, para variáveis contínuas e categóricas, respectivamente. Valores de $p<0,05$ foram considerados diferença estatisticamente significante. Resultados: Foram incluídos na análise 296 pacientes (186 com $<70$ anos e 110 com $>70$ anos). Não foi observada diferença em relação às características clínico-angiográficas entre os grupos e o sucesso angiográfico foi semelhante $(96,6 \%$ vs. $97,1 \% ; p=0,91)$. Ocorreu tendência a maior mortalidade $(1,81 \%$ vs. $0,53 \% ; p=0,06)$ e a maior incidência de acidente vascular encefálico $(1,81 \%$ vs. $0 \%$; $p=0,06)$ no grupo mais idoso. A incidência de cirurgia de urgência e de complicações vasculares não diferiu entre os grupos. Ocorreu maior incidência de nefropatia induzida pelo contraste no grupo $>70$ anos $(2,7 \%$ vs. $0 \%$; $p=0,02)$. Conclusão: Pacientes com idade $>70$ anos apresentaram incidência aumentada de nefropatia induzida pelo contraste e tendência a maior mortalidade e a maior ocorrência de acidente vascular encefálico.

DESCRITORES: Angioplastia transluminal percutânea coronária. Contenedores. Idoso.

1 CINECORS - Hospital Ernesto Dornelles - Porto Alegre, RS. Correspondência: Cristiano de Oliveira Cardoso. Rua Anita Garibaldi, 2381 - apto. 707 - Boa Vista - Porto Alegre, RS - CEP 90480-201 E-mail: co.cardoso@terra.com.br

Recebido em: 30/12/2007 • Aceito em: 15/2/2008

\section{SUMMARY}

Percutaneous Coronary Intervention in Patients Aged > 70 Years-Old: in-Hospital Outcomes in the Current Era

Introduction: Observational data from the 90 's demonstrates that elderly population has an increased risk when treated by percutaneous coronary intervention $(\mathrm{PCl})$. Objective: To evaluate in-hospital outcomes in patients aged $>70$ years old who underwent $\mathrm{PCl}$ in the current era. Methods: In a single center, from January/2005 to June/2007, a total of 296 patients who underwent $\mathrm{PCl}$ were included in this analysis. The presence of risk factors, angiographic characteristics and in-hospital outcomes were recorded and compared among patients < 70-yo $(\mathrm{n}=186)$ and > 70-yo $(\mathrm{n}=110)$ in a dedicated database. For comparison purposes, unpaired $t$ test and chi-square were used for continuous and dichotomous variables respectively. An alpha $<0.05$ was considered significant. Results: There was no difference between groups $<70-$ yo and $>70-y o$ regarding clinical presentation and the presence of risk factors for cardiovascular disease. Also, there was a homogenous distribution of treated vessels, angiographic pattern, coronary calcification and success rate $(96.6 \%$ in $>70$-yo group x $97.1 \%<70-$ yo, $p=0.91)$. Regarding complications, there was an increased rate of contrast induced nephropathy in the older group $(2.7 \% \mathrm{x}$ $0 \%, p=0.02)$ that required dialysis. Additionally, we observed a trend in higher mortality $(1.81 \% \times 0.53 \%, p=0.06)$ and neurological compromise $(1.81 \% \times 0 \%, p=0.06)$ in the elderly population. Vascular complication rates and urgent surgical revascularization were similar in both groups. Conclusion: Patients older and younger than 70-yo present similar clinical profile, angiographic characteristics and success rate. However, the incidence of contrast-induced nephropathy requiring dialysis, mortality and neurological compromise are higher in the older group.

DESCRIPTORS: Angioplasty, transluminal, percutaneous coronary. Stents. Aged. 
A população brasileira acima dos 70 anos de idade vem crescendo nos últimos anos. Dados do Instituto Brasileiro de Geografia e Estatística (IBGE) ${ }^{1}$, referentes ao censo demográfico de 2000, demonstram que naquele ano cerca de seis milhões de pessoas apresentavam idade igual ou superior a 70 anos. O aumento da população idosa tem sido observado mundialmente $^{2}$, sendo esperado aumento proporcional de procedimentos médicos em indivíduos dessa faixa etária.

Em pacientes com mais de 70 anos, a intervenção coronariana percutânea (ICP) é uma estratégia de revascularização miocárdica freqüentemente utilizada ${ }^{3,4}$. Dados observacionais da década de 90 sugerem que os pacientes idosos são um grupo de risco elevado para $\mathrm{ICP}^{5,6}$, evoluindo com maior mortalidade quando comparados aos indivíduos mais jovens ${ }^{4}$.

A introdução dos stents coronários, no entanto, modificou os resultados imediatos das ICPs. Como se sabe, houve redução substancial das taxas de oclusão aguda comparativamente à angioplastia com balão e melhora do sucesso técnico em decorrência da evolução dos materiais ${ }^{7,8}$. Além disso, com a monitorização intensiva dos níveis de anticoagulação, as complicações hemorrágicas também foram reduzidas ${ }^{9}$. Na literatura contemporânea e nacional, porém, poucos trabalhados têm avaliado os resultados clínicos nessa população. Assim, o objetivo do presente estudo foi avaliar os desfechos hospitalares em pacientes com idade superior a 70 anos submetidos a ICP.

\section{MÉTODO}

\section{Seleção da amostra}

Entre janeiro de 2005 e junho de 2007, 296 pacientes consecutivos foram submetidos a ICP em nosso serviço. Foram obtidas informações referentes a fatores de risco para doença cardiovascular, diagnóstico clínico, indicação do exame e detalhes técnicos da ICP para cada intervenção coronária, as quais foram inseridas em um banco de dados. Para evitar qualquer viés de seleção não foram aplicados critérios de exclusão e todos os pacientes tratados no período mencionado foram incluídos.

\section{Características analisadas}

Foram coletadas e analisadas informações contidas nas fichas referentes a idade, sexo, fatores de risco para doença cardiovascular (diabetes, hipertensão arterial, tabagismo, dislipidemia, história familiar), apresentação clínica, indicação da ICP, vaso tratado, características da lesão, índice de sucesso e complicações, como óbito, infarto agudo do miocárdio (IAM) pós-procedimento, acidente vascular encefálico, cirurgia de revascularização de emergência, perfuração e tamponamento cardíaco, pseudo-aneurisma, isquemia de membro inferior pós-procedimento, hematoma retroperitoneal, nefropatia induzida pelo contraste e necessidade de diálise.

\section{Intervenção coronariana percutânea}

As ICPs foram realizadas de acordo com as técnicas habituais e conforme orientações das diretrizes vigentes ${ }^{10-12}$.

Em resumo, os procedimentos foram realizados por via femoral ou radial e pré-dilatação e inibidores da glicoproteína IIb/IIla foram utilizados a critério do operador. Todos os pacientes receberam heparina nãofracionada no início do procedimento $(100 \mathrm{U} / \mathrm{kg}$ ), e doses adicionais foram administradas conforme necessário. Terapia antiplaquetária dupla foi utilizada em todos os pacientes (ácido acetilsalicílico 200 mg/dia associado a clopidogrel $300 \mathrm{mg}$ em dose de ataque e manutenção de $75 \mathrm{mg} / \mathrm{dia}$ ou ticlopidina $250 \mathrm{mg}$ a cada 12 horas). O tratamento antiplaquetário duplo foi prescrito por 30 dias e por pelo menos seis meses aos pacientes que receberam stent convencional e stent farmacológico, respectivamente.

\section{Definições e desfechos intra-hospitalares}

Sucesso angiográfico do procedimento foi definido quando obtida lesão residual $<20 \%$ e fluxo coronário TIMI III após implante do stent. A presença de fatores de risco (história familiar para cardiopatia isquêmica, diabetes, hipertensão arterial sistêmica, dislipidemia e tabagismo) foi determinada de acordo com as definições atuais ${ }^{13,14}$. A cirurgia realizada imediatamente após ICP em decorrência de complicação no procedimento foi considerada cirurgia de revascularização de urgência (CRMU). IAM foi diagnosticado na presença de angina, alteração eletrocardiográfica e/ou oclusão/ suboclusão documentada à angiografia. Os desfechos foram contabilizados dentro do período de internação hospitalar, sendo avaliados: sucesso angiográfico, óbito, IAM, CRMU e as outras complicações previamente mencionadas.

\section{Análise estatística}

Os pacientes foram divididos em dois grupos (idade inferior ou superior a 70 anos), sendo os resultados apresentados como média, desvio padrão e porcentual. Para comparação entre grupos, foram utilizados teste $t$ de Student, para variáveis contínuas com distribuição normal, e qui-quadrado, para variáveis categóricas. Valores de $p<0,05$ foram considerados diferença estatisticamente significante (teste bicaudal).

\section{RESULTADOS}

No período estudado, foram incluídos 296 pacientes, sendo $186(62,8 \%)$ com idade inferior a 70 anos e $110(37,2 \%)$ com idade superior a 70 anos. A maioria era do sexo masculino $(60,8 \%$ vs. $68,2 \%$; $p=0,54)$. Aproximadamente um quarto dos pacientes apresentava diabetes melito $(24,7 \%$ vs. $20 \%$; $p=0,45)$. Angina estável foi o quadro clínico mais freqüentemente detectado (55,9\% vs. 60\%; p =0,72) (Tabela 1). A 
Cardoso CO, et al. Intervenção Coronariana Percutânea em Pacientes com Mais de 70 Anos: Análise de Desfechos Intra-Hospitalares na Era Contemporânea. Rev Bras Cardiol Invas. 2008;16(1):64-69.

artéria descendente anterior foi o vaso mais tratado $(33,1 \%$ vs. $41,7 \% ; p=0,32)$, assim como as lesões mais complexas tipo B2/C (Tabela 2). O diâmetro dos stents utilizados foi de 3,11 $\pm 0,40 \mathrm{~mm}$, o comprimento foi de 18,4 $\pm 6,2 \mathrm{~mm}$ e foram implantados com pressão de 14,4 $\pm 2,6$ atm. No total, 310 stents foram implan-

TABELA 1

Características de base nos pacientes com idade inferior e superior a 70 anos

\begin{tabular}{lccc}
\hline Variáveis & $<\mathbf{7 0}$ anos $(\mathbf{n}=\mathbf{1 8 6})$ & $>\mathbf{7 0}$ anos $(\mathbf{n}=\mathbf{1 1 0})$ & $\mathbf{p}$ \\
\hline Idade (anos) & $58,3 \pm 7,7$ & $75,9 \pm 4,3$ & $<0,001$ \\
Sexo masculino (\%) & 60,8 & 68,2 & 0,54 \\
Diabetes melito (\%) & 24,7 & 20 & 0,45 \\
HAS (\%) & 54,8 & 50 & 0,65 \\
Tabagismo (\%) & 22 & 18,2 & 0,51 \\
Dislipidemia (\%) & 51,6 & 59,1 & 0,50 \\
História familiar para Cl (\%) & 45,2 & 38,2 & 0,45 \\
Quadro clínico & & & 0,19 \\
$\quad$ Isquemia silenciosa (\%) & 4,8 & 1,8 & 0,72 \\
$\quad$ Angina estável (\%) & 55,9 & 60 & 0,55 \\
SCA sem supradesnível de ST (\%) & 30 & 0,21 \\
SCA com supradesnível de ST (\%) & 25,8 & 8,2 & \\
\hline $\mathrm{n}=$ número de pacientes; HAS = hipertensão arterial sistêmica; Cl = cardiopatia isquêmica; SCA = síndrome coronariana aguda.
\end{tabular}

TABELA 2

Características angiográficas dos procedimentos percutâneos nos pacientes com idade inferior e superior a 70 anos

\begin{tabular}{lccc}
\hline Variáveis & $<\mathbf{7 0}$ anos & $>$ 70 anos & p \\
\hline Vaso tratado (\%) & & 1 & 0,31 \\
Tronco de coronária esquerda & 2,8 & 41,7 & 0,32 \\
Artéria descendente anterior & 33,1 & 3,9 & 0,34 \\
Ramo diagonal & 6,7 & 17,5 & 0,82 \\
Artéria circunflexa & 16,3 & 10,7 & 0,06 \\
Ramo marginal & 4,5 & 21,4 & 0,22 \\
Artéria coronária direita & 29,8 & 1 & 0,69 \\
Ramo descendente posterior & 0,6 & 2,9 & 0,31 \\
Enxerto de veia safena & 5,6 & 0 & 0,44 \\
Enxerto de artéria mamária & 0,6 & & 0,87 \\
Tipo de lesão* (\%) & 1,7 & 2 & 0,46 \\
A & 28,8 & 23,5 & 0,56 \\
B1 & 44,1 & 50 & 0,89 \\
B2 & 25,4 & 24,5 & 0,27 \\
C & 27,2 & 35,9 & 0,42 \\
Presença de cálcio (\%) & 13,5 & 10 & 0.95 \\
Presença de trombo (\%) & 34,1 & 33,6 & 0,73 \\
Ramos envolvidos (\%) & 21 & 19,1 & 0,91 \\
Lesão com comprimento $>$ 20 mm (\%) & $86,17 \pm 10,01$ & 0,87 \\
Estenose pré-procedimento (\%) & $2,89 \pm 0,56$ & 0,97 \\
Estenose pós-procedimento (\%) & 97,1 & \\
Sucesso angiográfico (\%) & $84,60 \pm 11,99$ & \\
\hline * Classificação do tipo de lesão de acordo com American Heart Association/American College of Cardiology & \\
\hline
\end{tabular}


tados: Driver (19\%), Cypher $(18,7 \%)$, Pro-Kinetic $(13,2 \%)$, Lekton $(10 \%)$, CoStar $(8,1 \%)$, Endeavor $(5,5 \%)$, RStent $(4,8 \%)$, Coroflex $(4,8 \%)$, Taxus $(4,2 \%)$ Liberte $(2,9 \%)$, Supralimus $(2,6 \%), 7$ Blue $(1,9 \%)$, Express $2(1 \%)$, Multilink (0,6\%), Crossflex (0,6\%), Genius-Magic (0,3\%), Infinnium $(0,3 \%)$, Penta $(0,3 \%)$, Bx-Velocity $(0,3 \%)$, Tenax $(0,3 \%)$ e JoStent $(0,3 \%)$. Os stents farmacológicos foram implantados em $38 \%$ dos procedimentos.

O índice de sucesso angiográfico foi alto e sem diferença entre os grupos (96,6\% vs. 97,1\%; $p=0,97)$, porém o grupo com idade superior a 70 anos apresentou tendência a maior incidência de óbito $(0,53 \%$ vs. $1,81 \% ; p=0,06)$ e acidente vascular encefálico $(0 \%$ vs. $1,81 \% ; p=0,06)$ (Figura 1). Não foi observado IAM pós-intervenção em nenhum dos grupos. O único caso de CRMU ocorreu no grupo com idade inferior a 70 anos, situação em que o paciente necessitou revascularização miocárdica por resultado subótimo da angioplastia.

O óbito no grupo com idade superior a 70 anos decorreu de perfuração coronária com posterior tamponamento cardíaco. Apesar de realizada drenagem pericárdica, esse paciente desenvolveu pneumonia e insuficiência renal, e faleceu durante a internação hospitalar. No segundo caso, o paciente apresentou quadro de acidente vascular encefálico pós-procedimento e faleceu em decorrência de complicações pulmonares. O único caso de óbito no grupo com idade inferior a 70 anos decorreu de IAM anterior extenso complicado por choque cardiogênico.

Insuficiência renal secundária a nefropatia induzida pelo contraste ocorreu mais freqüentemente nos idosos $(0 \%$ vs. $2,7 \%$; $p=0,02)$, dois terços dos quais necessitaram de diálise como forma de tratamento. Em relação às complicações vasculares, não foi observa-

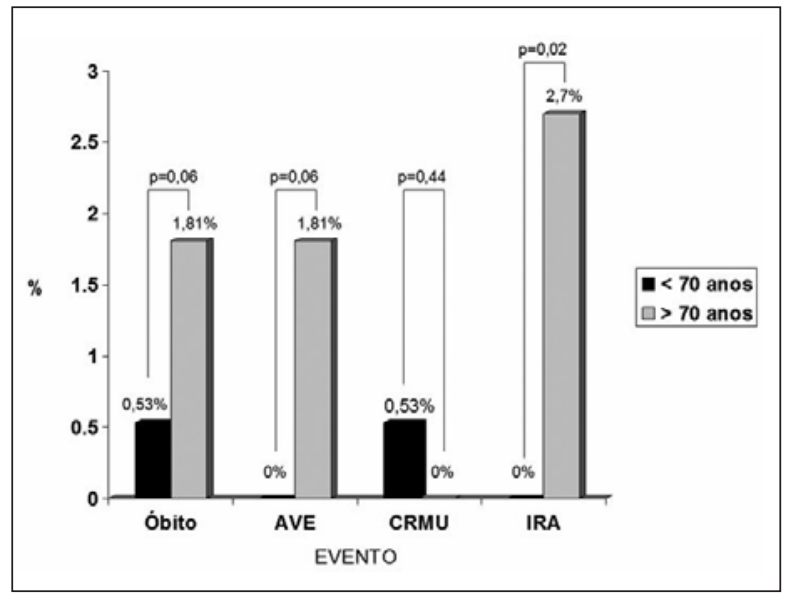

Figura 1 - Comparação entre os grupos de pacientes com idade inferior e superior a 70 anos.

$\mathrm{AVE}=$ acidente vascular encefálico; CRMU = cirurgia de revascularização miocárdica de urgência; IRA = insuficiência renal aguda. da diferença estatística entre os grupos na formação de pseudo-aneurisma $(1,61 \%$ vs. $0,9 \%$; $p=0,61)$, hematoma retroperitoneal ( $0 \%$ em ambos os grupos), isquemia de membro inferior pós-procedimento $(0 \%$ em ambos os grupos), perfuração coronária ( $0 \%$ vs. $0,9 \% ; p=0,19)$ e tamponamento cardíaco ( $0 \%$ vs. $0,9 \% ; p=0,19)$. A transfusão sanguínea não foi necessária em nenhum paciente e não foram observadas diferenças no que se refere às taxas de sangramento entre os portadores de síndrome isquêmica aguda com ou sem supradesnivelamento do segmento ST.

\section{DISCUSSÃo}

O presente estudo objetivou avaliar os desfechos hospitalares em pacientes com idade inferior e superior a 70 anos quando submetidos a ICP na era contemporânea. Dados observacionais da década de 90 demonstram que a população idosa tem características clínicas peculiares quando comparada à de pacientes mais jovens. Além da idade, essa população também apresenta prevalência aumentada de fatores de risco e maior morbidade cardiovascular. Isso determina à população com mais de 70 anos, que muitas vezes é excluída dos estudos clínicos, características especiais, fazendo com que seu tratamento seja diferente quando comparado ao da população de mais jovens. Em revisão das recomendações preconizadas pelas diretrizes atuais ${ }^{15}$, observa-se que os pacientes idosos não são tratados de acordo com as mesmas, sendo menor o uso de ácido acetilsalicílico e de betabloqueadores e a indicação de $\mathrm{ICP}^{16}$. Em nosso estudo, ambos os grupos estudados não apresentaram diferenças em relação aos fatores de risco e à indicação do procedimento. Embora a amostra seja pequena, os grupos foram comparáveis por apresentarem características semelhantes. Esse fenômeno, com prevalência aumentada de fatores de risco cardiovascular na população mais jovem e até mesmo em crianças, vem sendo observado nos dias de hoje e nosso estudo talvez seja representativo das mudanças acontecidas na distribuição dos fatores de risco na população $0^{17,18}$.

Nossa análise demonstra que tanto pacientes idosos quanto mais jovens podem se beneficiar da ICP, com altos índices de sucesso, fato que também tem sido observado por outros autores ${ }^{19,20}$. Atualmente, as ICPs têm desempenhado papel importante no tratamento da cardiopatia isquêmica, cujos índices de sucesso imediato com o uso de stents e com a melhora nos dispositivos utilizados são superiores a $90 \%$. Por isso, a idade não pode ser considerada um fator limitante aos procedimentos percutâneos. Se considerarmos o benefício a longo prazo nessa população, os resultados do Stent or Surgery Trial (estudo SoS) ${ }^{21}$, que comparou tratamento cirúrgico versus $\mathrm{ICP}$, demonstram que um ano após tratamento os pacientes com mais de 65 anos de idade se beneficiam tanto de tratamen- 
to clínico como de revascularização cirúrgica, sem vantagem de uma estratégia sobre outra. A ICP, portanto, deve ser considerada método efetivo de revascularização na população idosa, pois apresenta resultados imediatos satisfatórios e seguimento tardio favorável. Pfisterer et al. ${ }^{22}$ conduziram estudo randomizado comparando estratégia invasiva de revascularização e tratamento clínico otimizado, em pacientes com mais de 70 anos, e evidenciaram que essa população é suscetível a maior número de complicações relacionadas ao procedimento, seja por revascularização percutânea ou cirúrgica. No entanto, ao final de um ano, o grupo de pacientes inicialmente alocado para tratamento clínico apresentou $50 \%$ de chance de reinternação por angina ou necessidade de intervenção.

Apesar de os resultados angiográficos serem semelhantes em ambos os grupos, as complicações continuam mais freqüentes nos pacientes mais velhos. Em nosso estudo, a prevalência de insuficiência renal pósprocedimento decorrente da nefropatia induzida pelo contraste foi compatível com a da literatura, porém a necessidade de diálise foi superior. O risco de nefropatia induzida pelo contraste e a necessidade de diálise podem ser estimados por meio de escore de pontos, em que são atribuídos pontos a cada característica clínica $^{23}$. Considerando que a população idosa apresenta reduzida taxa de filtração glomerular, é de se presumir que a nefropatia induzida pelo contraste ocorreria mais freqüentemente nesse grupo de indivíduos. Embora esse evento possa ser previsto, a morbidade relacionada ainda é significativa, fazendo com que esse risco seja considerado na indicação do procedimento. Em casos de pacientes com alta probabilidade para nefropatia induzida pelo contraste, medidas profiláticas, como hidratação, uso de contraste de baixo peso molecular ${ }^{24}$ e medidas alternativas, como hidratação com bicarbonato ${ }^{25}$ e $\mathrm{N}$-acetilcisteína ${ }^{26,27}$, devem ser consideradas.

Adicionalmente às complicações renais, observouse tendência para maior mortalidade e ocorrência de acidente vascular encefálico no grupo idoso. Por se tratar de uma população que sabidamente apresenta índices mais altos de complicações, nossos achados são condizentes com os da literatura ${ }^{5,6}$. Com relação às complicações vasculares, não foi observada diferença entre a freqüência de hematoma retroperitoneal, isquemia aguda pós-intervenção e pseudo-aneurisma em ambos os grupos. Acreditamos que tal fato seja decorrente do cuidado com os acessos vasculares nos dias de hoje, com monitorização intensa dos níveis de anticoagulação, melhora do material utilizado e redução do calibre dos introdutores. A inexistência de IAM em ambos os grupos do estudo merece considerações: os critérios de definição são restritos e não ocorreu mensuração sistemática de enzimas cardíacas pós-intervenção. É possível que, pelos critérios assumidos, a real prevalência de IAM esteja subestimada.

\section{Limitações do estudo}

O presente estudo tem limitações que devem ser consideradas. Essa é uma análise retrospectiva em centro único e que apresenta amostra pequena de pacientes. Os desfechos restringem-se apenas à fase intra-hospitalar, o que limita qualquer conclusão com respeito à evolução tardia dos pacientes com idade superior ou inferior a 70 anos, uma vez que o estudo carece de seguimento a longo prazo.

\section{CONCLUSÃO}

Os pacientes com idade superior a 70 anos apresentaram perfil clínico, características angiográficas e taxa de sucesso semelhantes aos de pacientes mais jovens, porém com incidência aumentada de nefropatia induzida pelo contraste necessitando de diálise e tendência a maior mortalidade e ocorrência de acidente vascular encefálico.

\section{REFERÊNCIAS BIBLIOGRÁFICAS}

1. IBGE. Disponível em: http://www.ibge.gov.br/home/estatistica/ populacao/censo2000/primeiros_resultados_amostra/grandes_ regioes/pdf/tabela_2_7_8.pdf

2. Schwartz JB, Zipes DP. Cardiovascular disease in specials populations. In: Zipes DP, Libby P, Bonow RO, Braunwald E, eds. Braunwald's heart disease. $7^{\text {th }}$ ed. Philadelphia: Elsevier Saunders; 2005. p.1925-49.

3. Fraker TD Jr., Fihn SD, Gibbons RJ, Abrams J, Chatterjee K, Daley J, et al. 2007 chronic angina focused update of the ACC/AHA 2002 guidelines for the management of patients with chronic stable angina: a report of the American College of Cardiology/American Heart Association Task Force on Practice Guidelines Writing Group to develop the focused update of the 2002 guidelines for the management of patients with chronic stable angina. J Am Coll Cardiol. 2007;50(23):2264-74.

4. Alexander KP, Anstrom KJ, Muhlbaier LH, Grosswald RD, Smith PK, Jones RH, et al. Outcomes of cardiac surgery in patients $>$ or $=80$ years: results from the National Cardiovascular Network. J Am Coll Cardiol. 2000;35(3):731-8.

5. Thompson RC, Holmes DR Jr., Gersh BJ, Mock MB, Bailey KR. Percutaneous transluminal coronary angioplasty in the elderly: early and long-term results. J Am Coll Cardiol. $1991 ; 17(6): 1245-50$.

6. Wennberg DE, Makenka DJ, Sengupta A, Lucas FL, Vaitkus PT, Quinton $\mathrm{H}$, et al. Percutaneous transluminal coronary angioplasty in the elderly: epidemiology, clinical risk factors, and in-hospital outcomes. The Northern New England Cardiovascular Disease Study Group. Am Heart J. 1999;137(4 pt 1):639-45.

7. Roubin GS, Cannon AD, Agrawal SK, Macander PJ, Dean LS, Baxley WA, et al. Intracoronary stenting for acute and threatened closure complicating percutaneous transluminal coronary angioplasty. Circulation. 1992;85(3):916-27.

8. Hearn JA, King SB $3^{\text {rd }}$, Douglas JS Jr., Carlin SF, Lembo NJ, Ghazzal ZM. Clinical and angiographic outcomes after coronary artery stenting for acute or threatened closure after percutaneous transluminal coronary angioplasty. Initial results with a balloon-expandable, stainless steel design. Circulation. 1993;88(5 Pt 1):2086-96.

9. Kelsey SF, James M, Holubkov AL, Holubkov R, Cowley MJ, Detre KM. Results of percutaneous transluminal coronary 
angioplasty in women. 1985-1986 National Heart, Lung, and Blood Institute's Coronary Angioplasty Registry. Circulation. 1993;87(3):720-7.

10. King SB $3^{\text {rd }}$, Smith SC Jr., Hirshfeld JW Jr., Jacobs AK, Morrison DA, Williams DO, et al. 2007 Focused Update of the ACC/AHA/SCAI 2005 Guideline Update for Percutaneous Coronary Intervention: a report of the American College of Cardiology/American Heart Association Task Force on Practice Guidelines: 2007 Writing Group to Review New Evidence and Update the ACC/AHA/SCAI 2005 Guideline Update for Percutaneous Coronary Intervention, Writing on Behalf of the 2005 Writing Committee. Circulation. 2008;117(2):261-95.

11. Silber S, Albertsson P, Aviles FF, Camici PG, Colombo A, Hamm C, et al. Guidelines for percutaneous coronary interventions. The Task Force for Percutaneous Coronary Interventions of the European Society of Cardiology. Eur Heart J. 2005;26(8):804-47.

12. Smith SC Jr., Feldman TE, Hirshfeld JW Jr., Jacobs AK, Kern MJ, King SB $3^{\text {rd }}$, et al. ACC/AHA/SCAI 2005 guideline update for percutaneous coronary intervention: a report of the American College of Cardiology/American Heart Association Task Force on Practice Guidelines (ACC/AHA/SCAI Writing Committee to Update 2001 Guidelines for Percutaneous Coronary Intervention). Circulation. 2006;113(7): e166-286.

13. Graham I, Atar D, Borch-Johnsen K, Boysen G, Burell G, Cifkova R, et al. European guidelines on cardiovascular disease prevention in clinical practice: full text. Fourth Joint Task Force of the European Society of Cardiology and other societies on cardiovascular disease prevention in clinical practice (constituted by representatives of nine societies and by invited experts). Eur J Cardiovasc Prev Rehabil. 2007;14 Suppl 2:S1-113.

14. Hayman LL, Meininger JC, Daniels SR, McCrindle BW, Helden $\mathrm{L}$, Ross J, et al. Primary prevention of cardiovascular disease in nursing practice: focus on children and youth: a scientific statement from the American Heart Association Committee on Atherosclerosis, Hypertension, and Obesity in Youth of the Council on Cardiovascular Disease in the Young, Council on Cardiovascular Nursing, Council on Epidemiology and Prevention, and Council on Nutrition, Physical Activity, and Metabolism. Circulation. 2007;116(3): 344-57.

15. Ryan TJ, Bauman WB, Kennedy JW, Kereiakes DJ, King SB $3^{\text {rd }}$, McCallister BD, et al. Guidelines for percutaneous transluminal coronary angioplasty. A report of the American Heart Association/American College of Cardiology Task Force on Assessment of Diagnostic and Therapeutic Cardiovascular Procedures (Committee on Percutaneous Transluminal Coronary Angioplasty). Circulation. 1993;88(6):2987-3007.

16. Schoenenberger AW, Radovanovic D, Stauffer JC, Windecker
S, Urban P, Eberli FR, et al. Age-related differences in the use of guideline-recommended medical and interventional therapies for acute coronary syndromes: a cohort study. J Am Geriatr Soc. 2008

17. Prabhakaran P, Ajay VS, Prabhakaran D, Gottumukkala AK, Shrihari JS, Snehi U, et al. Global cardiovascular disease research survey. J Am Coll Cardiol. 2007;50(24):2322-8.

18. Shalitin S, Phillip M. Frequency of cardiovascular risk factors in obese children and adolescents referred to a tertiary care center in Israel. Horm Res. 2008;69(3):152-9.

19. Kobayashi Y, Mehran R, Mintz GS, Dangas G, Moussa I, Lansky AJ, et al. Comparison of in-hospital and one-year outcomes after multiple coronary arterial stenting in patients $>$ or $=80$ years old versus those $<80$ years old. Am J Cardiol. 2003;92(4):443-6.

20. Cohen HA, Williams DO, Holmes DR Jr., Selzer F, Kip KE, Johnston JM, et al. Impact of age on procedural and 1-year outcome in percutaneous transluminal coronary angioplasty: a report from the NHLBI Dynamic Registry. Am Heart J. 2003;146(3):513-9.

21. Zhang Z, Mahoney EM, Spertus JA, Booth J, Nugara F, Kolm $P$, et al. The impact of age on outcomes after coronary artery bypass surgery versus stent-assisted percutaneous coronary intervention: one-year results from the Stent or Surgery (SoS) trial. Am Heart J. 2006;152(6):1153-60.

22. Pfisterer $M$, Buser $P$, Osswald $S$, Allemann U, Amann W, Angehrn W, et al. Outcome of elderly patients with chronic symptomatic coronary artery disease with an invasive vs optimized medical treatment strategy: one-year results of the randomized TIME trial. JAMA. 2003;289(9):1117-23.

23. Mehran R, Aymong ED, Nikolsky E, Lasic Z, lakovou I, Fahy $M$, et al. A simple risk score for prediction of contrastinduced nephropathy after percutaneous coronary intervention: development and initial validation. J Am Coll Cardiol. 2004;44(7):1393-9.

24. McCullough PA, Bertrand ME, Brinker JA, Stacul F. A metaanalysis of the renal safety of isosmolar iodixanol compared with low-osmolar contrast media. J Am Coll Cardiol. 2006;48(4):692-9.

25. Briguori C, Airoldi F, D'Andrea D, Bonizzoni E, Morici N, Focaccio A, et al. Renal Insufficiency Following Contrast Media Administration Trial (REMEDIAL): a randomized comparison of 3 preventive strategies. Circulation. 2007;115(10): 1211-7.

26. Azmus AD, Gottschall C, Manica A, Manica J, Duro K, Frey $M$, et al. Effectiveness of acetylcysteine in prevention of contrast nephropathy. J Invasive Cardiol. 2005;17(2):80-4.

27. Marenzi G, Assanelli E, Marana I, Lauri G, Campodonico J, Grazi M, et al. N-acetylcysteine and contrast-induced nephropathy in primary angioplasty. N Engl J Med. 2006;354(26):2773-82. 